\title{
FORMALIZATION OF REGION OF DANGEROUS COURSES AND SPEEDS OF VESSELS AT EXTERNAL THEIR PROCESS CONTROL OF DIVERGENCE
}

\section{ФОРМАЛИЗАЦИЯ ОБЛАСТИ ОПАСНЫХ КУРСОВ И СКОРОСТЕЙ СУДОВ ПРИ ВНЕШНЕМ УПРАВЛЕНИИ ИХ ПРОЦЕССОМ РАСХОЖДЕНИЯ}

\author{
I.A. Burmaka, $P h D$, associate professor, S.S. Pasechnyuk, $P h D$ student \\ И.А. Бурмака, к.т.н., дочент, С.С. Пасечнюк, аспирант \\ National University «Odessa Maritime Academy», Ukraine \\ Наџиональный университет «Одесская морская академия», Украина
}

\begin{abstract}
The method of forming of impermissible region of courses of one ship and speeds of other ship is offered for estimation of no unconcern of situation of rapprochement and choice of safe maneuver of divergence. A case is considered, when speed of ship which changes direction, more large to speed of other ship, that maneuvers.

The region of impermissible courses of one ship and speeds of other ship is considered in this case, at united which distance of the shortest rapprochement more small on a size from maximum - possible distance, that rapprochement of ships is dangerous. It is considered principle of forming of such region, coming that from that its border is formed by points which meet condition of equality distance of the shortest rapprochement and maximum - possible distance.

It is shown that on the intervals of change of course of the first ship, which can be larger direct corner, the values of speed of the second ship for both scopes grow droningly. In work there is the got graphic interpretation of the offered region of impermissible courses of one ship and speeds of other ship for the case of the considered correlation of speeds of ships which are dangerously drawn together. The mentioned region is contained between two scopes which do not intersect. Points between scopes answer courses and speeds of ships, at which distance of the shortest rapprochement of more small after maximum - possible distance, that characterizes dangerous rapprochement of ships. Therefore, if a point with the initial parameters of motion of ships is between the first and second scopes, that it belongs to the region of impermissible courses of one ship and speeds of other ship, rapprochement of ships is dangerous. At that rate for safe divergence of ships it is needed to choose the parameters of deviation of ships, that course and speed thus, that a point proper to them was on the border of region of impermissible courses of one ship and speeds of other ship the nearest to the initial point and distance between the mentioned points was minimum.

Thus, in work analytical expressions are got for the calculation of scopes of region of impermissible courses of one ship and speeds of other ship, that is dangerously
\end{abstract}


drawn together, and also the offered procedure of estimation of unconcern of rapprochement.

Keywords: safety of navigation, maneuver of divergence of vessels by the change of course and speed, impermissible region of parameters of motion of vessels.

\section{PEФEPAT}

Запропоновано спосіб формування недопустимої області курсів одного судна і швидкостей іншого судна для оцінки небезпечності ситуації зближення та вибору безпечного маневру розходження. Розглянуто випадок, коли швидкість судна, яке міняє курс, більша швидкості іншого судна, що маневрує.

В даному випадку розглянуто область недопустимих курсів одного судна $\mathrm{i}$ швидкостей іншого судна, при поєднані яких дистанція найкоротшого зближення менша по величині від гранично - допустимої дистанції, тобто зближення суден являється небезпечним. Розглянуто принцип формування такої області, виходячи з того, що ії межу утворюють точки, які задовольняють умові рівності дистанції найкоротшого зближення та гранично - допустимої дистанції.

Показано, що на інтервалах зміни курсу першого судна, які можуть бути більші прямого кута, значення швидкості другого судна для обох меж монотонно зростають. В роботі одержана графічна інтерпретація запропонованої області недопустимих курсів одного судна і швидкостей іншого судна для випадку розглянутого співвідношення швидкостей суден, які небезпечно зближуються. Згадана область міститься між двома межами, які не перетинаються. Точки між межами відповідають курсам і швидкостям суден, при яких дистанція найкоротшого зближення менша за гранично - допустиму дистанцію, що характеризує небезпечне зближення суден. Тому, якщо точка 3 початковими параметрами руху суден знаходиться між першою Ошибка! Ошибка связи.і другою межами, тобто належить області недопустимих курсів одного судна і швидкостей іншого судна, то зближення суден небезпечне. В такому разі для безпечного розходження суден потрібно вибрати параметри ухилення суден, тобто курсу і швидкості таким чином, щоб відповідна їм точка знаходилась на найближчій до початкової точки межі області недопустимих курсів одного судна і швидкостей іншого судна і відстань між згаданими точками була мінімальною.

Таким чином, в роботі одержано аналітичні вирази для розрахунку меж області недопустимих курсів одного судна і швидкостей іншого судна, що небезпечно зближуються, а також запропонована процедура оцінки безпечності зближення.

Ключові слова: безпечність судноводіння, маневр розходження зміною курсу і швидкості, неприпустима область параметрів руху суден. 
Постановка проблемы в общем виде и ее связь с важными научными или практическими задачами

Обеспечение безопасности судовождения при плавании судов в стесненных водах является важнейшей проблемой повышения безопасности мореплавания. В настоящее время стесненные районы плавания с особенно интенсивным движением оборудуются станциями управления движением судов, назначением которых является контроль процесса судовождения и управления движением опасно сближающихся судов. В связи с этим разработка способов управления опасно сближающимися судами является актуальным научным направлением.

Анализ последних достижений и публикаций, в которых начато решение данной проблемы и выделение нерешенных ранее частей общей проблемы

Принцип внешнего управления группой судов при возникновении ситуации опасного сближения рассмотрен в работе [1], а работы [2, 3] посвящены вопросам применения опасных областей параметров движения судов для их безопасного расхождения, причем в работе [2] рассмотрено использование опасной области курсов двух судов для выбора допустимого маневра расхождения, а в работе [3] предложен маневр расхождения судов изменением их скоростей.

\section{Формулировка целей статьи}

Целью данной статьи является формализация способа формирования области курсов одного судна и скоростей другого судна для оценки опасности ситуации сближения и выбора безопасного маневра расхождения.

\section{Изложение основного материала исследования с обоснованием полученных научных результатов}

В работе [2] рассмотрен способ формирования области опасных курсов пары сближающихся судов, с помощью которого возможна оценка степени опасности ситуации сближения и выбора маневра расхождения судов изменением их курсов при постоянных скоростях. Способ расчета границ области опасных скоростей, позволяющий определить маневр расхождения судов изменением их скоростей при неизменных курсах, предложен в работе [3]. Однако возможности безопасного расхождения опасно сближающихся судов возрастают при использовании еще одного типа расхождения, при котором одно из судов изменяет курс, сохраняя неизменной скорость, а второе судно на постоянном курсе может снижать свою скорость. В указанном случае необходимо рассмотреть область опасных курсов одного судна и скоростей другого судна, при сочетании которых дистанция кратчайшего сближения меньше предельно-допустимой дистанции, т. е. сближение судов является опасным. Рассмотрим принцип формирования такой области, которую обозначим $\Omega_{\mathrm{KV}}$. Очевидно, что границу области $\Omega_{\mathrm{KV}}$ на плоскости $\mathrm{K}_{1} \times \mathrm{V}_{2}$ составляют точки $\left(\mathrm{K}_{1}, \mathrm{~V}_{2}\right)$, удовлетворяющие условию $\min \mathrm{D}\left(\mathrm{K}_{1}, \mathrm{~V}_{2}\right)=\mathrm{d}_{\mathrm{d}}$. 
Аналитически это выражается следующим образом. Из выражения для $\min \mathrm{D}[4]$ получим:

$$
\mathrm{K}_{\mathrm{ot}}=\alpha \mp \arcsin \left(\frac{\mathrm{d}_{\mathrm{d}}}{\mathrm{D}}\right) \text {, }
$$

поэтому справедливо равенство:

$$
\operatorname{tg} K_{o t}=\operatorname{tg}\left[\alpha \pm \arcsin \left(\frac{d_{d}}{D}\right)\right]
$$

Обозначаем $\gamma^{(1,2)}=\alpha \mp \arcsin \left(\frac{\mathrm{d}_{\mathrm{d}}}{\mathrm{D}}\right)$, следовательно,

Учитывая [4],

$$
\operatorname{tg} K_{\text {ot }}=\operatorname{tg} \gamma^{(1,2)}
$$

$$
\begin{aligned}
\operatorname{tg} \mathrm{K}_{\mathrm{ot}}= & \frac{\mathrm{V}_{1} \sin \mathrm{K}_{1}-\mathrm{V}_{2} \sin \mathrm{K}_{2}}{\mathrm{~V}_{1} \cos \mathrm{K}_{1}-\mathrm{V}_{2} \cos \mathrm{K}_{2}}, \quad \text { получим } \\
& \frac{\mathrm{V}_{1} \sin \mathrm{K}_{1}-\mathrm{V}_{2} \sin \mathrm{K}_{2}}{\mathrm{~V}_{1} \cos \mathrm{K}_{1}-\mathrm{V}_{2} \cos \mathrm{K}_{2}}=\frac{\sin \gamma^{(1,2)}}{\cos \gamma^{(1,2)}},
\end{aligned}
$$

откуда записываем зависимость между курсом одного судна $\mathrm{K}_{1}$ и скоростью другого судна $\mathrm{V}_{2}$, удовлетворяющим условию $\min \mathrm{D}=\mathrm{d}_{\mathrm{d}}$.

Выражение (1) принимает следующий вид:

$$
\begin{gathered}
\sin \mathrm{K}_{1} \cos \gamma^{(1,2)}-\cos \mathrm{K}_{1} \sin \gamma^{(1,2)}=\frac{\mathrm{V}_{2}}{\mathrm{~V}_{1}}\left(\sin \mathrm{K}_{2} \cos \gamma^{(1,2)}-\cos \mathrm{K}_{2} \sin \gamma^{(1,2)}\right), \quad \text { или } \\
\sin \left(\mathrm{K}_{1}-\gamma^{(1,2)}\right)=\frac{\sin \left(\mathrm{K}_{2}-\gamma^{(1,2)}\right)}{\mathrm{V}_{1}} \mathrm{~V}_{2} .
\end{gathered}
$$

Если обозначить $\mu^{(1,2)}=\frac{\sin \left(\mathrm{K}_{2}-\gamma^{(1,2)}\right)}{\mathrm{V}_{1}}$, то $\mathrm{V}_{2}^{(1,2)}=\frac{\sin \left(\mathrm{K}_{1}-\gamma^{(1,2)}\right)}{\mu^{(1,2)}}$.

Следовательно, существует две границы, на которых достигается равенство $\min \mathrm{D}=\mathrm{d}_{\mathrm{d}}$ :

$$
\begin{aligned}
& \mathrm{V}_{2}^{(1)}=\frac{\sin \left(\mathrm{K}_{1}-\gamma^{(1)}\right)}{\mu^{(1)}}=\frac{\mathrm{V}_{1}}{\sin \left[\mathrm{K}_{2}-\left(\alpha+\arcsin \frac{\mathrm{d}_{\mathrm{d}}}{\mathrm{D}}\right)\right]} \sin \left[\mathrm{K}_{1}-\left(\alpha-\arcsin \frac{\mathrm{d}_{\mathrm{d}}}{\mathrm{D}}\right)\right] \\
& \mathrm{V}_{2}^{(2)}=\frac{\sin \left(\mathrm{K}_{1}-\gamma^{(2)}\right)}{\mu^{(2)}}=\frac{\mathrm{V}_{1}}{\sin \left[\mathrm{K}_{2}-\left(\alpha-\arcsin \frac{\mathrm{d}_{\mathrm{d}}}{\mathrm{D}}\right)\right]} \sin \left[\mathrm{K}_{1}-\left(\alpha+\arcsin \frac{\mathrm{d}_{\mathrm{d}}}{\mathrm{D}}\right)\right]
\end{aligned}
$$


Так как рассматриваем изменение скорости торможением, то значения скоростей $\mathrm{V}_{2}^{(1,2)}$ должны удовлетворять условию $\mathrm{V}_{2 \mathrm{n}}>\mathrm{V}_{2}^{(1,2)} \geq 0$, где $\mathrm{V}_{2 \mathrm{n}}$ начальная скорость судна.

Рассмотрим, какие значения курса $\mathrm{K}_{1}$ соответствуют граничным значениям 0 и $\mathrm{V}_{2 \mathrm{n}}$ скорости $\mathrm{V}_{2}^{(1,2)}$. Прежде всего, отмечаем, что границы не могут быть определены для ситуации, когда $\mathrm{K}_{2}=\alpha \pm \arcsin \frac{\mathrm{d}_{\mathrm{d}}}{\mathrm{D}}$. Очевидно, из уравнений границ (2):

$$
\mathrm{K}_{1}\left(\mathrm{~V}_{2}^{(1,2)}=0\right)=\alpha \mp \arcsin \frac{\mathrm{d}_{\mathrm{d}}}{\mathrm{D}}=\gamma^{(1,2)}
$$

Для определения второго значения $\mathrm{K}_{1}\left(\mathrm{~V}_{2}^{(1,2)}=\mathrm{V}_{2 \mathrm{n}}\right)$ рассмотрим уравнение:

$$
\begin{gathered}
\mathrm{V}_{2}^{(1,2)}=\frac{\sin \left(\mathrm{K}_{1}-\gamma^{(1,2)}\right)}{\mu^{(1,2)}} \text { и подставляем } \mathrm{V}_{2}^{(1,2)}=\mathrm{V}_{2 \mathrm{n}} \\
\mathrm{V}_{2 \mathrm{n}}=\frac{\sin \left(\mathrm{K}_{1}-\gamma^{(1,2)}\right)}{\mu^{(1,2)}} \text {, откуда } \\
\sin \left(\mathrm{K}_{1}-\gamma^{(1,2)}\right)=\frac{\mathrm{V}_{2 \mathrm{n}}}{\mathrm{V}_{1}} \sin \left(\mathrm{K}_{2}-\gamma^{(1,2)}\right) \text {, поэтому } \\
\mathrm{K}_{1}=\gamma^{(1,2)}+\arcsin \left[\frac{\mathrm{V}_{2 \mathrm{n}}}{\mathrm{V}_{1}} \sin \left(\mathrm{K}_{2}-\gamma^{(1,2)}\right)\right], \text { или } \\
\mathrm{K}_{1}\left(\mathrm{~V}_{2}^{(1,2)}=\mathrm{V}_{2 \mathrm{n}}\right)=\gamma^{(1,2)}+\arcsin \left[\frac{\mathrm{V}_{2 \mathrm{n}}}{\mathrm{V}_{1}} \sin \left(\mathrm{K}_{2}-\gamma^{(1,2)}\right)\right]
\end{gathered}
$$

В случае $\mathrm{V}_{1}>\mathrm{V}_{2 \mathrm{n}}$ существуют значения $\mathrm{K}_{1}\left(\mathrm{~V}_{2}^{(1,2)}=\mathrm{V}_{2 \mathrm{n}}\right)$ для обеих границ, а если $\mathrm{V}_{1}<\mathrm{V}_{2 \mathrm{n}}$, то необходимо учитывать соотношения между величиной $\gamma^{(1,2)}$ и экстремальными относительными курсами $\mathrm{K}_{\mathrm{otmax}}$ и $\mathrm{K}_{\mathrm{otmin}}$.

Рассмотрим случай, когда $\mathrm{V}_{1}>\mathrm{V}_{2 \mathrm{n}}$ и находим граничные значения курса первого судна:

$$
\begin{gathered}
\mathrm{K}_{1}\left(\mathrm{~V}_{2}^{(1)}=0\right)=\alpha-\arcsin \frac{\mathrm{d}_{\mathrm{d}}}{\mathrm{D}}=\gamma^{(1)} ; \\
\mathrm{K}_{1}\left(\mathrm{~V}_{2}^{(2)}=0\right)=\alpha+\arcsin \frac{\mathrm{d}_{\mathrm{d}}}{\mathrm{D}}=\gamma^{(2)} ; \\
\mathrm{K}_{1}\left(\mathrm{~V}_{2}^{(1)}=\mathrm{V}_{2 \mathrm{n}}\right)=\gamma^{(1)}+\arcsin \left[\frac{\mathrm{V}_{2 \mathrm{n}}}{\mathrm{V}_{1}} \sin \left(\mathrm{K}_{2}-\gamma^{(1)}\right)\right] ;
\end{gathered}
$$




$$
\mathrm{K}_{1}\left(\mathrm{~V}_{2}^{(2)}=\mathrm{V}_{2 \mathrm{n}}\right)=\gamma^{(2)}+\arcsin \left[\frac{\mathrm{V}_{2 \mathrm{n}}}{\mathrm{V}_{1}} \sin \left(\mathrm{K}_{2}-\gamma^{(2)}\right)\right]
$$

Введем обозначения:

$$
\begin{gathered}
\mathrm{K}_{1 \min }^{(1)}=\mathrm{K}_{1}\left(\mathrm{~V}_{2}^{(1)}=0\right), \mathrm{K}_{1 \min }^{(2)}=\mathrm{K}_{1}\left(\mathrm{~V}_{2}^{(2)}=0\right) \\
\mathrm{K}_{1 \mathrm{max}}^{(1)}=\mathrm{K}_{1}\left(\mathrm{~V}_{2}^{(1)}=\mathrm{V}_{2 \mathrm{n}}\right), \mathrm{K}_{1 \max }^{(2)}=\mathrm{K}_{1}\left(\mathrm{~V}_{2}^{(2)}=\mathrm{V}_{2 \mathrm{n}}\right) .
\end{gathered}
$$

С учетом принятых обозначений:

$$
\begin{gathered}
\mathrm{K}_{1 \min }^{(1)}=\alpha-\arcsin \frac{\mathrm{d}_{\mathrm{d}}}{\mathrm{D}}=\gamma^{(1)}, \\
\mathrm{K}_{1 \mathrm{~min}}^{(2)}=\alpha+\arcsin \frac{\mathrm{d}_{\mathrm{d}}}{\mathrm{D}}=\gamma^{(2)}, \\
\mathrm{K}_{1 \max }^{(1)}=\gamma^{(1)}+\arcsin \left[\frac{\mathrm{V}_{2 \mathrm{n}}}{\mathrm{V}_{1}} \sin \left(\mathrm{K}_{2}-\gamma^{(1)}\right)\right], \\
\mathrm{K}_{1 \text { max }}^{(2)}=\gamma^{(2)}+\arcsin \left[\frac{\mathrm{V}_{2 \mathrm{n}}}{\mathrm{V}_{1}} \sin \left(\mathrm{K}_{2}-\gamma^{(2)}\right)\right] .
\end{gathered}
$$

Обращаем внимание, что изменение скорости второго судна $\mathrm{V}_{2}$ на участке $\mathrm{V}_{2} \in\left(0, \mathrm{~V}_{2 \mathrm{n}}\right)$ для первой границы происходит на участке курсов $\mathrm{K}_{1} \in\left(\mathrm{K}_{1 \min }^{(1)}, \mathrm{K}_{1 \max }^{(1)}\right)$, т. е. на интервале $\Delta \mathrm{K}_{1}^{(1)}=\mathrm{K}_{1 \max }^{(1)}-\mathrm{K}_{1 \min }^{(1)}$ или с учетом полученных выражений $\Delta \mathrm{K}_{1}^{(1)}=\arcsin \left[\frac{\mathrm{V}_{2 \mathrm{n}}}{\mathrm{V}_{1}} \sin \left(\mathrm{K}_{2}-\gamma^{(1)}\right)\right]$. Аналогично для второй границы: $\mathrm{K}_{1} \in\left(\mathrm{K}_{1 \mathrm{~min}}^{(2)}, \mathrm{K}_{1 \max }^{(2)}\right) \quad$ и $\quad \Delta \mathrm{K}_{1}^{(2)}=\arcsin \left[\frac{\mathrm{V}_{2 \mathrm{n}}}{\mathrm{V}_{1}} \sin \left(\mathrm{K}_{2}-\gamma^{(2)}\right)\right]$. Заметим, что интервалы $\Delta \mathrm{K}_{1}^{(1)}$ и $\Delta \mathrm{K}_{1}^{(2)}$ меньше $\pi / 2$, поэтому на этих интервалах значение скорости $\mathrm{V}_{2}$ для обеих границ монотонно возрастает. С учетом полученных результатов область $\Omega_{\mathrm{KV}}$ опасных параметров курса одного судна и скорости второго судна, заключенная между первой $\mathrm{G}_{1 \mathrm{KV}}$ и второй $\mathrm{G}_{2 \mathrm{KV}}$ границами для случая $\mathrm{V}_{1}>\mathrm{V}_{2 \mathrm{n}}$ выглядит, как показано на рис. 1. Поэтому, если точка с начальными параметрами движения судов

$\mathrm{M}_{\mathrm{n}}\left(\mathrm{K}_{\mathrm{n} 1}, \mathrm{~V}_{2 \mathrm{n}}\right)$ находится между первой ошибка! Ошибка связи. и второй $\mathrm{G}_{2 \mathrm{KV}}$ границами, т. е. $\left(\mathrm{K}_{\mathrm{n} 1}, \mathrm{~V}_{2 \mathrm{n}}\right) \in \Omega_{\mathrm{KV}}$, то имеет место неравенство $\min \mathrm{D}\left(\mathrm{K}_{\mathrm{n} 1}, \mathrm{~V}_{2 \mathrm{n}}\right)<\mathrm{d}_{\mathrm{d}}$, и сближение судов является опасным. В этом случае необходимо выбрать параметры уклонения судов $\mathrm{K}_{1 \mathrm{y}}$ и $\mathrm{V}_{2 \mathrm{y}}$ таким образом, чтобы соответствующая им точка $\mathrm{M}_{\mathrm{y}}\left(\mathrm{K}_{1 \mathrm{y}}, \mathrm{V}_{2 \mathrm{y}}\right)$ находилась на ближайшей к точке $\mathrm{M}_{\mathrm{n}}\left(\mathrm{K}_{\mathrm{n} 1}, \mathrm{~V}_{2 \mathrm{n}}\right)$ границе области $\Omega_{\mathrm{KV}}$ и расстояние между точками $\mathrm{M}_{\mathrm{n}}$ и M было минимальным, как показано на рис. 1. 


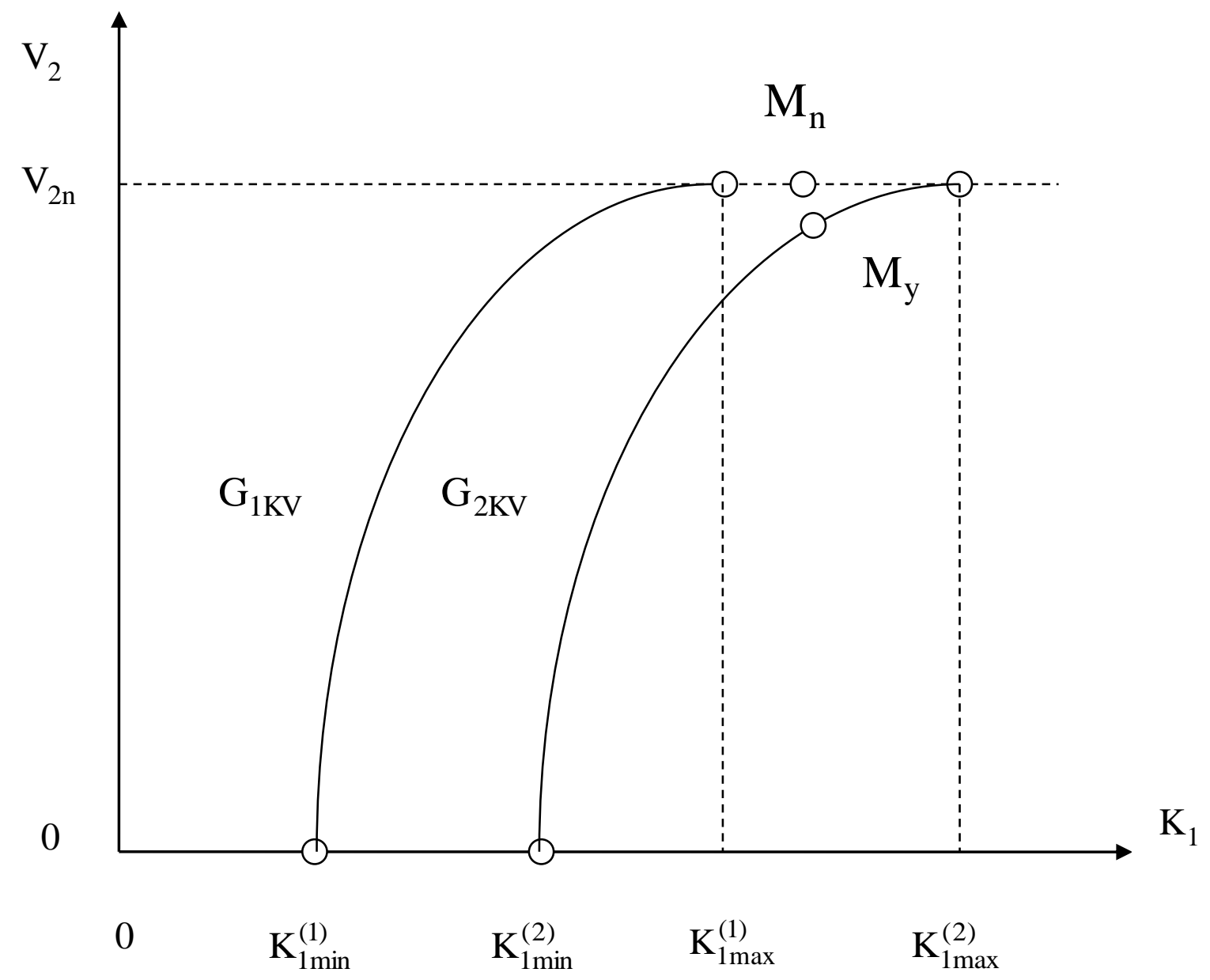

Рис. 1. Область $\Omega_{\mathrm{KV}}$ опасных параметров судов

Выводы и перспектива дальнейшей работы по данному направлению

Таким образом, в данной статье предложен способ формирования области курсов одного судна и скоростей другого судна для оценки опасности ситуации сближения и выбора безопасного маневра расхождения. В дальнейшем целесообразно рассмотреть ситуацию, когда скорость судна, изменяющего курс, больше скорости своего партнера по опасному сближению.

\section{ЛИТЕРАТУРА}

1. Бурмака И.А., Калиниченко Г.Е., Кулаков М.А. Основные характеристики группы судов при внешнем управлении процессом судовождения// Судовождение: Сб. научн. трудов / ОНМА, Вып. 26. - Одесса: «ИздатИнформ», 2016 - С. 35-40.

2. Булгаков А.Ю. Использование опасной области курсов двух судов для выбора допустимого маневра расхождения/ Булгаков А.Ю.// Водный транспорт. - 2014. №2 (20). - С. 12 - 17. 
3. Кулаков М. А. Процедура определения маневра расхождения изменением скоростей судов // Судовождение: Сб. научн. трудов / ОНМА, Вып. 27. Одесса: «ИздатИнформ», 2016 - С. 112-118.

4. Бурмака И.А. Управление судами в ситуации опасного сближения / И.А Бурмака., Э.Н Пятаков., А.Ю. Булгаков - LAP LAMBERT Academic Publishing, - Саарбрюккен (Германия), -2016. - 585 с. 